(e-migrinter

e-Migrinter

9| 2012

Immigrés, illégaux, réfugiés. Questions sur les enquêtes et les catégories

\title{
Monique Bertrand, De Bamako à Accra : mobilités urbaines et ancrages locaux en Afrique de l'Ouest
}

\section{Kévin Mary}

\section{OpenEdition}

\section{Journals}

Édition électronique

URL : https://journals.openedition.org/e-migrinter/786

DOI : 10.4000/e-migrinter.786

ISSN : 1961-9685

Éditeur

UMR 7301 - Migrinter

Édition imprimée

Date de publication : 20 juillet 2012

Pagination : 113-115

ISSN : 1961-9685

Référence électronique

Kévin Mary, « Monique Bertrand, De Bamako à Accra : mobilités urbaines et ancrages locaux en Afrique de l'Ouest », e-Migrinter [En ligne], 9 | 2012, mis en ligne le 19 juillet 2016, consulté le 20 mai 2021. URL : http://journals.openedition.org/e-migrinter/786 ; DOI : https://doi.org/10.4000/e-migrinter.786 


\section{Bertrand, Monique (2011) De Bamako à Accra : mobilités urbaines et ancrages locaux en Afrique de l'Ouest, Paris, Karthala, 376 p.}

Kévin Mary

$\mathbf{M}$ onique Bertrand présente ici les travaux réalisés dans le cadre de son habilitation à diriger des recherches. Cet ouvrage est donc le fruit de ses enquêtes menées en Afrique depuis une vingtaine d'années, au cours desquelles l'auteure a élargi son champ d'investigation de l'Afrique francophone (Bamako, au Mali) à l'Afrique anglophone (Accra, au Ghana), dans la lignée du nombre croissant de chercheurs français qui s'autorisent à sortir du « pré carré » francophone et s'ouvrent sur les pays anglophones africains ${ }^{1}$.

Le texte se présente d'abord comme une comparaison entre ces deux métropoles ouest africaines. Cette approche permet de souligner la diversité des Afriques et des villes africaines qui s'avère encore trop peu mise en avant dans les travaux de recherche actuels. Monique Bertrand insiste à ce titre sur la différence entre les héritages coloniaux qui influencent les structures foncières urbaines maliennes et ghanéennes. Le texte apparait très dense et offre différents niveaux de lecture. Monique Bertrand, dans une approche multiscalaire qui articule la « ville d'en haut (...) et la ville d'en bas » (p. 177), fait néanmoins de la question foncière le fil conducteur de son propos (p. 26).

La première partie de l'ouvrage pose les bases de la comparaison (chapitre 1) et fournit des informations méthodologiques (chapitre 2), très détaillées, à l'usage des chercheurs travaillant sur les "Suds", dans des contextes où l'informel domine et dans lesquels les sources écrites (statistiques et

${ }^{1}$ Voir par exemple les travaux de Philippe GervaisLambony (1994, 2009) et Véronique Lassailly-Jacob (2001) sur l'Afrique Australe ou encore ceux d'Amandine Spire sur le Ghana (2011). archives notamment) manquent cruellement ou sont incomplètes lorsqu'elles existent. Monique Bertrand parle ainsi de la « richesse des sources pauvres» (p. 49) qui, une fois agrémentées de notes informelles recueillies dans l'ambiance des bureaux administratifs, arrivent à donner un sens plus général aux phénomènes étudiés. L'auteure évoque des notes constituées d'«anecdotes emblématiques» (p. 51), ou encore de «bribes de conversations retranscrites du bambara entre deux verres de thé et une tournée d'arachides» (p. 53). C'est ce qui conduit Monique Bertrand à militer pour une " micro-geografia » ${ }^{2}$ (p. 84) qui suggère d'étudier la ville au «microscope», dans la lignée de travaux menés il y a déjà plus de vingt ans et publiés dans un numéro spécial des Cabiers d'Études africaines ${ }^{3}$. Il s'agit d'une approche dont l'auteure indique qu'elle est plus à même de saisir « la respiration sociale intime [de ces métropoles], au fil de convivialités et de conflictualités de quartiers» (p. 84). Monique Bertrand nous apprend également que le corpus constitué dans cet ouvrage est d'ordre à la fois qualitatif et quantitatif, basé notamment sur des enquêtes longitudinales à passages répétés durant plusieurs années dans les concessions familiales de Bamako et d'Accra. Cet usage du matériau quantitatif (en l'occurrence l'administration de questionnaires) en Afrique est assez rare pour être souligné et constitue l'un des apports majeur de cet ouvrage.

Monique Bertrand décrit ensuite les pratiques résidentielles et gestionnaires de la ville. Les chercheurs travaillant sur les

${ }^{2} \mathrm{Ou}$ géographie au microscope.

${ }^{3}$ Villes africaines au microscope (1981), Cabiers d'Études africaines, vol. XXI (1-3), n 81-83. 
dynamiques familiales en Afrique pourront lire au chapitre 4 une discussion sur le concept de «ménage» dans les villes africaines. L'auteure a opté pour une définition qui va au-delà de celle des recensements officiels malien et ghanéen qui se fondent uniquement sur les liens de parenté avec le «chef de famille». Dans cet ouvrage, la notion de ménage est élargie aux liens avec les "épouses", mais également à d'autres types d'agencements domestiques: parents éloignés, alliés, enfant adoptifs, etc. Il en résulte une définition des ménages en quatre cercles domestiques: noyau familial, dépendants familiaux, dépendants sociaux et économiques. S'en suit une réflexion stimulante sur la création de la ville "par le haut »: celle des politiques et des projets menés par les bailleurs de fonds internationaux, mais également celle des élites municipales (chapitre 5). Le niveau d'analyse des élites met en lumière des politiques urbaines au service de carrières politiques locales (p. 182) et apporte d'intéressants éléments explicatifs sur les stratégies employées par les élites qui sont localement jugées à leur capacité à s'adapter aux appels d'offres internationaux pour acquérir des positions de pouvoir politique. L'auteure précise que ces stratégies s'avèrent pourtant fragiles au Mali, où les élus municipaux «dépassent rarement un mandat » (p. 182) et où l'on constate un fort renouvellement de ces élites. Cet aspect met en lumière des processus paradoxaux de formation des élites au Mali, où les stratégies qui permettent l'accession au pouvoir n'aboutissent pas à sa reproduction.

La dernière partie de l'ouvrage adopte une approche "par le bas », via les pratiques citadines, que l'auteure interroge à travers la tension entre ancrages et mobilités (chapitres 6 et 7). On y découvre des locataires pauvres, ballottés d'un côté à l'autre de ces agglomérations, ayant du mal à s'organiser collectivement et qui demeurent souvent oubliés des programmes de luttes contre la pauvreté (p. 292). À l'inverse de ces « cadets sociaux », l'ancrage résidentiel, qui suppose la mobilisation d'un capital à la fois économique et social (p. 261), confère un véritable statut d'« aîné ». L'auteure souligne que l'acquisition de ce statut d'«ainé » se constitue également par l'intermédiaire des mobilisations d'épargnes migratoires investies dans le domaine foncier (chapitre 7), qui réactivent cette tension entre ancrage et mobilité selon une opposition entre populations locales et migrants internationaux. L'ouvrage se termine par une réflexion sur la "modernité» africaine (chapitre 8), dans la lignée des travaux de Jean Copans ${ }^{4}$ (1990) qui, lui aussi, traite des différences entres les Afriques francophones et anglophones.

Par son approche critique des politiques urbaines (chapitre 5) et par la manière dont elle nous invite à décentrer notre regard sur les villes africaines et leur « modernité ", mais aussi à se détacher des modes scientifiques du moment (p. 327), cet ouvrage peut être considéré comme une recherche de géographie sociale, telle que pratiquée par une partie des géographes français. Cette approche critique, couplée avec une réelle sensibilité pour la «question sociale », devrait donc interpeller ce courant de la géographie, notamment autour des réflexions sur «l'espace social» (chapitre 8). L'auteure souligne en particulier les incohérences de la vision des bailleurs de fonds internationaux - voire des chercheurs à l'encontre des habitants les plus pauvres de ces villes, tour à tour stigmatisés puis mis en avant sous l'égide de la "promotion du local », dans leurs projets urbains successifs. Monique Bertrand $y$ décèle «une représentation tronquée de la pauvreté, amputée de l'analyse de la richesse et de sa redistribution $»$ (p. 198).

Au final, l'ouvrage de Monique Bertrand frappe d'abord par la qualité de son

${ }^{4}$ Copans, Jean, 1990, La longue marche de la modernité africaine. Savoirs, intellectuels, démocratie, Paris, Karthala, $406 \mathrm{p}$. 
analyse, qui parvient à ne jamais se couper $\mathrm{du}$ «terrain». On pourra simplement regretter la relative faiblesse de la production cartographique. Mais de cette grande connaissance empirique et théorique résulte un ouvrage très riche, qui fournit au lecteur d'utiles clés à la compréhension de ces deux métropoles ouest-africaines.

Kevin Mary

Doctorant en géographie MIGRINTER - UMR 7301

ESO - UMR 6590

CNRS / Universités de Poitiers et Caen

kevin.mary@unicaen.fr 